\title{
CAD e BIM: Evolução ou Revolução na Aprovação de Projetos de Edificações nas Instâncias Legais?
}

\author{
CAD and BIM: Evolution or Revolution in Building Projects Approval in Legal Instances?
}

\author{
Alexandre Monteiro de Menezes \\ Universidade FUMEC, Brasil \\ amenezes@fumec.br \\ Maria de Lourdes Silva Viana \\ Universidade FUMEC, Brasil \\ malu@fumec.br
}

\author{
Mário Lucio Pereira Junior \\ Universidade FUMEC, Brasil \\ mariopereira@fumec.br \\ Sérgio Ricardo Palhares
Universidade FUMEC, Brasil
palhares@fumec.br
}

\begin{abstract}
This research compared CAD and BIM, seeking to identify evolution, or revolution, in the field of building projects, focusing on municipal approval. The research sought to understand municipal, state and federal normative to approve architectural projects and studied CAD and BIM in the light of the technical documents generation submitted for building projects evaluation and approval. The aim was to compare the efficiency of 3D model for the analysis of projects in municipalities. The results identify adequacies and inadequacies on both platforms, but also formulate suggestions for improvements in the process of evaluation and approval of projects by local governments.
\end{abstract}

Keywords: BIM; Architectural design; Projects approval; Graphic communication; Buildings construction.

\section{Introdução}

Esta pesquisa investiga e compara o uso das tecnologias CAD (Computer Aided Design) e BIM (Building Information Modeling), especialmente na fase de aprovação de projetos nas instâncias legais, com vistas a conhecer o estado da arte no cenário no município de Belo Horizonte/MG. Pesquisas anteriores indicam subutilização da tecnologia CAD e um ruído na comunicação entre os profissionais parceiros, bem como adequações e inadequações do uso da tecnologia BIM nas diversas etapas de projetos de edificações. Buscam-se identificar aspectos que caracterizam uma evolução, assim como aspectos que permitem falar em revolução no campo da prática profissional.

A hipótese da pesquisa acena para uma revisão nas atuais normas legais de aprovação de projetos de arquitetura, junto aos órgãos competentes, considerando-se o potencial das novas tecnologias, particularmente a tecnologia BIM, que operam com informações e geometria em 3D. A confirmação desta hipótese irá subsidiar desdobramentos para futuras pesquisas, bem como a necessidade de revisões das normativas atuais de aprovação de projetos de arquitetura junto ao poder público municipal.

A pesquisa se desenvolveu de forma qualitativa. Foi feita uma revisão da literatura que buscou investigar e possibilitar a compreensão de um cenário sobre as normativas municipais, estaduais e federais que regem os processos de aprovação de projetos de arquitetura junto aos órgãos competentes. A pesquisa investigou também as plataformas CAD e BIM à luz da geração de documentos técnicos que são encaminhados às instâncias de poder, com base nas ferramentas disponíveis nos softwares. Foi analisado um projeto de arquitetura efetivamente aprovado pela prefeitura Municipal de Belo Horizonte, desenvolvido pela plataforma CAD. Com base na revisão da literatura, foi verificada a adequação do software às normativas da prefeitura, quando da geração dos documentos técnicos em formato bidimensional.

O mesmo projeto de arquitetura foi desenvolvido em plataforma BIM, que gera documentação técnica a partir de modelos tridimensionais, com o objetivo de produzir ao final a mesma documentação necessária à aprovação na prefeitura Municipal de Belo Horizonte. Nesta operação, as ferramentas usadas para conversão das informações e modelos 3D, lógica do BIM, para documentos técnicos $2 \mathrm{D}$, lógica das normas atuais da prefeitura municipal, foram analisadas também com base na revisão da literatura.

O resultado das análises dos projetos de arquitetura desenvolvidos pelo CAD e pelo BIM para atenderem à mesma normativa de aprovação junto ao órgão competente, gerou condições de comparação entre as duas plataformas.

\section{Revisão da Literatura}

Os processos de projeto na indústria da construção civil no Brasil ainda são, em sua maioria, desenvolvidos com o uso de tecnologia 2D. Entretanto, existem iniciativas isoladas utilizando ferramentas CAD 3D ou BIM e várias pesquisas têm sido desenvolvidas nessa área, pois, a necessidade da melhoria da qualidade da obra e a 
redução de desperdício de material clamam por meios de elaboração do projeto de forma que as interferências sejam resolvidas antes da execução. Birx (2001), citado por Oliveira e Pereira (2011), estima que em menos de uma década aconteça a transição da plataforma CAD para a BIM, definida por Eastman et al (2008) como um conjunto de associações de processos voltados para a produção da obra, comunicação e análise do modelo do edifício.

No entanto, a ferramenta BIM ainda não é amplamente conhecida e tampouco um modelo estabelecido na maioria dos escritórios de arquitetura, o que demonstra que nem todos estão preparados para migrar para a nova plataforma (OLIVEIRA e PEREIRA, 2011). Em relação ao Brasil, Andrade e Ruschel (2009), citados por Oliveira e Pereira (2011), afirmam que a tecnologia BIM é um tema que ainda pode ser considerado atual em âmbito brasileiro. São de 2007 as primeiras pesquisas que citam a terminologia BIM. Desta época até hoje, verifica-se certa diversidade de trabalhos em BIM no Brasil, porém mais direcionados à gestão de projetos, ao trabalho colaborativo e a distância ou ainda aos sistemas colaborativos voltados para a construção civil.

Esses trabalhos explanatórios divulgam potencialidades da tecnologia ligadas a conceitos básicos. Oliveira e Pereira (2011) e Nardelli et al (2010) investigaram o estado da arte da tecnologia digital em escritórios de arquitetura paulistanos, adotando um modelo proposto por Oxman em 2006. Souza (2009) estudou o uso da ferramenta BIM em 13 escritórios de arquitetura brasileiros, realizando estudos de caso com ênfase na análise das influências da tecnologia no processo de projeto de arquitetura. A pesquisadora reforça a necessidade de avanços em termos de integração de projetos. A interpretação quantitativa gerou números pouco representativos ao se comparar o universo pesquisado frente ao contexto mais amplo. Ainda assim, o trabalho foi de extrema relevância e o único com o foco semelhante desta pesquisa dentre os coletados.

De acordo com Ferreira (2007) e Souza et al (2009) apud Goes (2011), o uso da plataforma BIM ainda se encontra restrito aos escritórios de arquitetura. É consenso que a tecnologia poderia ser utilizada para facilitar a compatibilização de projetos, auxiliando na redução de erros e facilitando as soluções de projeto. Entretanto, o modelo BIM está sendo usado mais como ferramenta de concepção e visualização do que de desenvolvimento e coordenação de projetos. As ferramentas BIM funcionam como facilitadoras do processo de projeto dentro do escritório de arquitetura, mas ainda não atingiram diretamente outros processos ligados à produção do edifício, enquanto o processo de compatibilização na verdade ainda ocorre conforme os moldes tradicionais do CAD.

Um estudo de caso foi desenvolvido por Goes (2011) com o objetivo de comparar o processo de compatibilização, utilizando uma ferramenta BIM com o procedimento tradicional bidimensional, simulando o processo de construção de um modelo de informação virtual completo. Para isso, foi escolhido um projeto já compatibilizado anteriormente, com registros de incompatibilidades e interferências. Os modelos de informação foram desenvolvidos separadamente e contemplavam projetos de arquitetura, estrutura, sistemas hidráulicos e de ar condicionado de um pavimento tipo para cada etapa de projeto, para posteriormente serem agrupados e integrar um único modelo. As interferências encontradas foram relatadas, classificadas e analisadas de maneira qualitativa e quantitativa.

A probabilidade de o modelo BIM ser caracterizado por informação inequívoca e consistente torna-se maior do que em CAD geométrico, pois representa a natureza do objeto. E como o BIM faz a consistência das informações, pois se trata de um modelo virtual. Os arquivos CAD geométricos são formados por elemento geométricos básicos, sem qualquer referência, cabendo ao projetista interpretar e dar significado às linhas e demais elementos (AYRES e SCHEER, 2007).

Goes (2011) afirma que essas inconsistências geométricas encontradas a partir da modelagem em BIM não haviam sido encontradas pelo processo convencional, o que pode ser explicado pelas limitações da representação bidimensional em relação ao desenvolvimento de projeto. A modelagem tridimensional BIM não só possibilita melhor visualização do projeto, mas seu próprio processo de desenvolvimento permite a detecção de conflitos relativos à falta e à incoerência de informações, quando houver.

Segundo Crespo e Ruschiel (2007) citados por Hilgenberg (2011), na prática arquitetônica deve-se prever a adequação da ferramenta proposta, utilizando todo o seu potencial. A diferença entre um modelo BIM e um modelo 3D convencional é que este último é apenas uma representação tridimensional geométrica do edifício, enquanto um modelo BIM é organizado como um protótipo do prédio, em termos de pisos do edifício, espaços, paredes, portas, janelas, entre outros elementos, e uma vasta gama de informações associadas a cada um desses componentes, através de relações paramétricas.

O modelo BIM pode normalmente ser visto em 3D, mas também inclui informações usadas por outros aplicativos de análise, tais como estimativa de custos, simulação de consumo de energia, iluminação natural, etc. (GOES, 2011) Importante ressaltar que um processo BIM pressupõe o envolvimento de vários integrantes ao longo de todo o ciclo de vida da edificação (Addor et al, 2010 e Santos, 2009), além da comunicação entre os vários sistemas de análise do modelo tridimensional. Sendo assim, interoperabilidade é um conceito importante, sendo a condição básica para que os modelos conversem entre si.

No entanto, segundo Eastman et al, citados por Oliveira e Pereira (2011), o nível de comprometimento é uma das barreiras para a implementação de novas tecnologias. Esforços individuais não chegam a atingir o principal objetivo do BIM, que é a integração absoluta do projeto e dos membros de suas equipes com o mundo real. O importante é uma sensibilização coletiva para adoção de sistemas compatíveis. Segundo Hilgenberg e outros (2012), o mercado precisa demonstrar maturidade organizacional e possuir metodologias de trabalho compatíveis.

De acordo com Addor et al (2010), quando a utilização de BIM for uma realidade, dentro do ambiente AEC (Arquitetura, Engenharia e Construção), ao longo de toda o processo do projeto, os atuais índices de erros deverão cair significativamente, pois o uso pleno dessa tecnologia permite análise, simulação e extração de dados do modelo, possibilitando ganhos de confiabilidade para as informações geradas durante todo o processo. Isso se deve ao fato 
das informações estarem concentradas em um único modelo, sendo todos os documentos possíveis extraídos dele e qualquer alteração será refletida em todos os documentos. (ADDOR et al, 2010).

A interação de toda a cadeia produtiva AEC é fundamental para o sucesso da implantação do sistema, envolvendo desde os projetistas, passando por incorporadoras e construtoras, poder público, setor de obras/construção, setor de vendas e indústria, uma vez que o processo permeia todos os setores e pressupõe esta integração. (ADDOR et al, 2010).

Segundo Kvan (2000) apud Scheer e Ayres Filho (2009), a construção de edifícios é uma atividade multidisciplinar que depende da cooperação de vários profissionais. As diferentes disciplinas envolvidas produzem soluções que são completas apenas em relação ao conjunto de técnicas da própria disciplina, isoladamente. Considerando a informação necessária para a realização da totalidade do processo construtivo, é preciso integrar essas soluções parciais, momento no qual podem surgir conflitos entre elas.

Kalay (2005), citado neste mesmo artigo de Scheer e Ayres Filho (2009), afirma que gerenciar a resolução destes conflitos torna-se cada vez mais difícil, porque o conhecimento técnico é cada vez mais complexo e ao mesmo tempo segmentado em diferentes profissões. Ainda de acordo com Scheer e Ayres Filho (2009), apesar dos recentes desenvolvimentos, uma série de desafios relacionados às práticas projetuais correntes ainda precisa ser superada para que esse tipo de integração torne-se usual na indústria da construção.

Outro grande desafio é a questão da proteção da informação inserida no modelo, que estando disponível para todos os outros envolvidos, acaba diluindo a noção de responsabilidade técnica. Citando o órgão norte-americano National Building Information Modeling Standard, os autores ressaltam que a organização em torno da plataforma BIM demanda formas seguras para controlar o uso da informação contida no modelo, incluindo níveis de acesso, controle de versões, certificação de autoria e origem da documentação legal gerada a partir do modelo, como projetos para aprovação, listas de materiais para contratação ou mesmo quantitativos para licitações.

Souza, Amorin e Lyrio (2009) acreditam que poderia ser criado um padrão a ser utilizado por todos os escritórios, fornecido pelas empresas que desenvolvem os softwares. Este padrão deve ser construído de forma cooperativa, com a participação de toda cadeia produtiva. De acordo com os autores, os principais motivos apontados para busca de utilização da tecnologia BIM dizem respeito à diminuição de erros de projeto e aumento de qualidade. Segundo eles, os aspectos legais relacionados ao BIM merecem destaque, e é preciso que se busquem soluções legais para atribuição do pertencimento do modelo e da responsabilidade na exatidão do conteúdo de informações.

No que diz respeito à aprovação de projetos por prefeituras, Scheer e Romero (2009), realizaram pesquisa que investigou, com auxílio da tecnologia BIM, "como garantir agilidade nos procedimentos das aprovações de projetos de edificações, estabelecendo a aplicação de um método de estudo de campo, tendo como objeto a Prefeitura Municipal de Curitiba." Os autores comparam o processo de aprovação de um projeto apresentado na forma tradicional e a análise do mesmo projeto com informações em uma estrutura de dados da plataforma BIM.

Foram identificadas as eficiências e deficiências das duas formas de apresentação do projeto e, também, formuladas sugestões de melhorias no processo de verificação de projetos pela prefeitura e apresentadas recomendações sobre a possibilidade do uso da nova tecnologia. Foram levantadas as principais barreiras no que diz respeito ao processo de aprovação de projetos, tendo sido apontada como mais relevante a falta de interação entre profissionais e as interpretações erradas em relação à legislação.

Ao se constatar que grande parte dos profissionais nunca teve contato com algum software BIM, evidencia-se a necessidade da disseminação deste conceito e das facilidades que ele pode oferecer ao profissional da AEC. Tais facilidades podem ser apontadas através da melhoria de visualização do objeto modelado e do aumento da produtividade, integrando informações de diversos projetos num mesmo modelo digital (HILGENBERG e outros 2011).

Costa e Rêgo (2010) salientam que o investimento em ferramentas BIM tem crescido, em parte em função da propaganda feita pelos revendedores, classificando um programa BIM como um "novo" CAD. Isso evidencia uma compreensão incorreta das funções da plataforma BIM, que implica na adoção da mesma maneira de projetação, não enfatizando a relação entre os envolvidos no processo, a necessidade do gerenciamento do desenvolvimento do projeto e, quase sempre, na necessidade da reorganização da própria empresa.

\section{Metodologia}

Esta pesquisa se configura a partir do desdobramento de pesquisas anteriores, todas desenvolvidas na Universidade FUMEC, por Menezes e outros, em 2008, 2009, 2010 (MENEZES e OUTROS 2008, 2009, 2010). Os procedimentos metodológicos iniciam-se a partir da leitura de pesquisas anteriores, anais de congressos, material pesquisado na web e referências bibliográficas resgatadas em artigos, teses e dissertações. Esta leitura enfatizou os conteúdos que subsidiavam as análises daqueles resultados que se aproximaram do objetivo da pesquisa corrente.

A revisão da literatura evidenciou os processos de aprovação de projetos legais de arquitetura junto ao poder público, com base na representação gráfica dos mesmos por meio das plataformas CAD e BIM. Também foram evidenciados os parâmetros aplicados para avaliação de projetos de arquitetura pela Prefeitura Municipal de Belo Horizonte.

Em seguida buscou-se mapear escritórios de arquitetura em Belo Horizonte que já tivessem aprovado projetos legais desenvolvida na plataforma BIM. Interessou investigar qual material gráfico foi gerado e apresentado aos examinadores da prefeitura, e também como se deu o processo de aprovação. Em seguida, foi escolhido um projeto de arquitetura desenvolvido na plataforma CAD, por um dos arquitetos pesquisadores, que havia sido aprovado pela prefeitura municipal de Belo Horizonte nos últimos seis meses. Paralelamente, o mesmo projeto foi desenvolvido na plataforma 
BIM. Na medida em que o grupo possuía um mesmo projeto desenvolvido em ambas as plataformas, foi marcada uma nova entrevista, agora com arquitetas examinadoras da prefeitura municipal de Belo Horizonte.

Nesta entrevista, foi apresentado o produto desenvolvido nas duas plataformas. Objetivou-se entender, do ponto de vista das examinadoras, em qual delas haveria melhores condições técnicas de análise dos parâmetros de aprovação da Lei de Uso e Ocupação do Solo. Com base na análise das entrevistas realizadas, foi possível sistematizar as informações. A análise destas informações explicitou, para diversos parâmetros aplicados na avaliação de projetos de arquitetura, como as duas plataformas respondem com maior ou menor eficiência no sentido de prover o examinador de melhor condição de análise técnica do projeto.

Para aferir as eficiências das plataformas para análise de parâmetros urbanísticos, foram eleitos o cálculo de área permeável, avaliação do afastamento lateral e do gabarito do edifício e análise das modificações empreendidas no edifício. Estes foram desenvolvidos nas plataformas CAD e BIM e, posteriormente, os resultados gráficos foram comparados e analisados de modo a evidenciar quais resultados davam mais subsídio ao examinador na análise dos parâmetros observados.

\section{Resultados}

A entrevista com as funcionárias do setor de avaliação e aprovação de projetos da Prefeitura Municipal de Belo Horizonte teve como objetivo identificar a melhor maneira de visualizar alguns parâmetros utilizados no processo de análise e aprovação de projetos de edificações. A hipótese era de que o modelo 3D desenvolvido na plataforma BIM possivelmente trouxesse uma maior eficiência da visualização para a análise dos projetos de edificações, em função da melhor visualização das informações disponíveis.

Os dados levantados apontam para uma revisão nas normais legais atuais de aprovação de projetos de arquitetura, junto aos órgãos competentes. A expectativa era de que o modelo tridimensional fosse mais eficiente para a visualização dos parâmetros de análise. Uma das entrevistadas demonstrou resistência à introdução de novas tecnologias, alegando que o desenho em papel é mais vantajoso para o registro do projeto da edificação junto a cartórios, proprietário e órgãos municipais. A avaliadora apontou o possível alto custo do software como empecilho para a adoção de um programa, pois seriam necessárias várias licenças. Foi ponderado que qualquer cidadão deve ter fácil acesso ao projeto sem a necessidade de um programa específico de computador.

A pesquisa entende que o registro é importante, mas ele pode ser feito de outra forma, por meio de arquivos eletrônicos não modificáveis. Nos programas gráficos atuais, qualquer documento pode ser facilmente gerado e existem visualizadores gratuitos que poderiam ser disponibilizados pela prefeitura, assim como existem equipamentos à disposição nos tribunais de justiça. A própria prefeitura microfilma os documentos, mostrando confiança em novas tecnologias.

Houve indícios de comparação das plataformas 2D e 3D, no que concerne à eficiência para fornecimento do material gráfico. Tanto nas ferramentas 2D quanto nas 3D, a informação está disponível, mas com o modelo tridimensional nenhum cálculo é necessário e seria bem mais fácil a conferência dos itens de avaliação no modelo 3D.

Para objetos complexos, parece que a visualização 3D facilita e torna mais rápida a análise. Esta pesquisa ressalta que há uma legislação a ser cumprida e o profissional deveria ter a responsabilidade de segui-la e, se comprovada a eficiência da análise por meio do modelo 3D, que seria desejável que esta conclusão provocasse uma revisão das normas visando a melhoria da qualidade de vida na cidade, partindo do pressuposto de que os parâmetros atualmente avaliados priorizam elementos de interesse coletivo. A evolução dos softwares possibilita a definição dos parâmetros de modo que o próprio software acuse a incompatibilidade do projeto com os limites impostos pela legislação.

Durante a entrevista com as funcionárias da $\mathrm{PMBH}$, verificou-se que, apesar do compreensível apego ao método tradicional de exame que atende à demanda atual, com o esclarecimento das dúvidas e com a visualização, no modelo, dos parâmetros examinados, os técnicos passaram a acenar positivamente com a possível contribuição da ferramenta para o processo.

O que evidenciou a contribuição positiva foi o fato do modelo 3D não permitir maquiagem, evitando que sejam elaborados somente cortes que não mostrem informações que burlem a legislação, escondendo possíveis imperfeições. Com o modelo tridimensional não existe o risco de uma representação simplificada, que induziria a uma análise menos abrangente, na medida em que todas as informações técnicas do projeto estão contidas na representação 3D e possibilitam aferir qualquer parâmetro técnico desejado, para fins de análise, independentemente daqueles oficialmente normatizados pelo órgão público.

\section{Conclusão}

As conclusões desta pesquisa parecem coincidir com resultados apontados por outras pesquisas relevantes na área (SCHEER e ROMERO 2009; SOUZA, AMORIN e LYRIO 2009; HILGENBERG e OUTROS 2011). Este estudo possibilitou identificar adequações e inadequações em ambas as formas de apresentação do projeto, como também indicar a necessidade de melhoria no processo de aprovação de projetos pela prefeitura de Belo Horizonte.

Constatou-se com o estudo, que parte dos profissionais da $A E C$ nunca teve contato e desconhece os softwares BIM, evidenciandose a necessidade da maior divulgação e disseminação deste conceito e das facilidades que ele pode oferecer aos profissionais. Tais facilidades podem ser apontadas através da integração das informações de diversos projetos num mesmo modelo digital, da melhoria de visualização do objeto modelado e do aumento da produtividade.

Esses resultados parecem dar apoio à opinião de Souza, Amorin e Lyrio (2009) quando afirmam que a visualização 3D permite melhorar o entendimento do projeto pelos envolvidos, facilita as soluções de projeto como também a análise dos profissionais dos setores de aprovação de projetos nas prefeituras municipais.

Alguns problemas foram apontados nas entrevistas com relação ao uso de software BIM para avaliação de projetos em prefeituras: 
o alto custo do software; a necessidade de alguma forma de registro, para o cartório e proprietários; o impacto na comunidade profissional ligada à edificação, e o difícil acesso pela população em geral. Atualmente os softwares BIM apresentam realmente um custo elevado para aquisição, quando comparados aos programas tradicionais de escritório. Este custo, de fato, poderia inviabilizar a utilização pelo poder público.

Entretanto, existe a possibilidade da utilização de visualizadores gratuitos, como já acontece com vários tipos de programas de computação gráfica. Assim, parece não ser interesse dos desenvolvedores de software cobrar daqueles que irão visualizar e extrair informações dos produtos gerados, mas sim daqueles que de fato irão construir os modelos, no caso Arquitetos e Engenheiros.

O software REVIT da Autodesk pode ser instalado sem a compra da licença e, após os 30 dias de teste, pode permanecer instalado, mantendo-se funcional para análise e avaliação do modelo, apresentando restrições para salvar e imprimir. Dessa forma, as prefeituras podem utilizar, legalmente, o programa para avaliar o modelo, sem custo. O registro documental sempre foi importante e tradicional no armazenamento das informações.

A pesquisa conclui que, se a prefeitura continuar a realizar os exames da maneira atual, a plataforma BIM parece ser desnecessária, ou seja, fica confirmada a hipótese inicial da necessidade de revisão dos procedimentos de aprovação de projeto pela prefeitura. Como sugestão de uso do modelo, a prefeitura poderia solicitar que o profissional o entregasse com um invólucro que facilitasse a visualização dos parâmetros urbanísticos exigidos pela legislação, para a aprovação do projeto.

\section{Agradecimentos}

Gostaríamos de agradecer à Universidade FUMEC e à FUNADESP pelo apoio financeiro e logístico.

\section{Referências}

Addor, M. R. A. et al. Colocando o "i" no BIM. Revista acadêmica arq.urb, no4. Universidade São Judas Tadeu, São Paulo. 2010. Disponível em: http://www.usjt.br/arq.urb/numero_04/arqurb4_06_miriam.pdf Acesso em: 26 set. 2012.

Eastman, C., Teicholz, P., Sacks, R., Liston, K. BIM. Handbook: A Guide to Building Information Modeling for Owners, Managers, Designers, Engineers and Contractors, ed. John Wiley \& Sons, New Jersey, 2008.

Goes, R. H. T. B. Compatibilização de projetos: comparação entre o BIM e CAD 2D. In: V TECNOLOGIAS DA INFORMAÇÃO E COMUNICAÇÃO TIC, 2011, Salvador, Anais eletrônicos, 1 CD-ROM

Hilgenberg, F. B. et al. Uso de BIM pelos profissionais de arquitetura em Curitiba. Revista científica Gestão e Tecnologia de Projetos, São Paulo, v.7, n.1, jan. 2011. Disponível em: http://www.iau.usp.br/posgrad/gestaodeprojetos/index.php/gestao deprojetos/article/view/196, Acesso em: 10 set. 2012.

Hippert, M. A. S., Araújo, T. T. Análise e representação em contextos diversos: projeto, técnica e gestão do ambiente construído. In: I Encontro Nacional da Associação de Pesquisa e Pós-Graduação Em
Arquitetura e Urbanismo, 29 de novembro a 03 de dezembro de 2010, Rio de Janeiro. Anais eletrônicos. Disponível em: http://www.anparq.org.br/dvd-enanparq/simposios/173/173-739-1SP.pdf, Acesso em 09 set. 2012.

Menezes, A. M. ; Palhares, S. R. ; Pereira Junior, M. L. ; Viana, M. L. S., Comunicação Gráfica entre profissionais parceiros no projeto de edifícios na era digital. In: COBENGE XXXVI congresso Brasileiro de Educação em Engenharia, 2008, São Paulo.

Anais eletrônicos, CD-ROM.

.Procedimentos para a qualidade na comunicação gráfica igital entre profissionais parceiros no projeto de edificações. In: VIII International Conference on Graphics Engineering for Arts and Design - XIX Simpósio Nacional de Geometria descritiva e DesenhoTécnico, 2009, Bauru/SP. Graphica' 09 Linguagem e Estratégias da Expressão Gráfica: Comunicação e Conhecimento. Bauru: UNESP, 2009. v. 1.

.A adequação (ou não) dos aplicativos BIM às teorias contemporâneas de ensino de projeto de edificações. In: XIV Congreso de la Sociedad Iberoamericana de gráfica Digital - SIGRADI 2010, 2010, Bogotá. SIGRADI 2010. Bogotá : Ediciones Uniandes, 2010. v. 1. p. 55-57.

Nardelli, E. S., Vincent, C. C. Pesquisa revela como escritórios de arquitetura utilizam softwares. AU Arquitetura e Urbanismo, São Paulo, 2009. Disponível em: http://www.revistaau.com.br/ arquitetura- urbanismo/ 188 /imprime 155941.asp>. Acesso em: 30 out. 2012

Oliveira, L. C., Pereira, A. T. C.. O uso de tecnologias BIM em escritórios de Arquitetura relacionado ao modo de implantação. In: V Encontro de Tecnologia de Informação e Comunicação na Construção, Salvador, Bahia, Brasil, 4 e 5 de agosto de 2011

- Mudanças metodológicas decorrentes da implantação recente de BIM em escritórios de arquitetura. In: XV CONGRESO DE LA SOCIEDAD IBEROAMERICANA DE GRÁFICA DIGITAL - SIGRADI 2011, Santa Fé, Argentina, 16-18 de novembro de 2011.

Disponível em: $<$ http://cumincades.scix.net/data/works/att/sigradi2011_287.content. pdf> Acesso em: 05 set. 2012.

Santos, E. T. Building Information Modeling você realmente sabe o que é? In: VII Workshop Brasileiro de Gestão do Processo de Projetos na Construção de Edifícios WBGPPCE, Curitiba, 2007, Disponível em: <http://www.cesec.ufpr.br/workshop2007/PDF/0702\%20Building\%2 OInformation\%20Modelin g\%20-\%20Toledo\%20Santos.pdf>. Acesso em: 04 jul. 2009.

Scheer, S., Ayres Filho, C. G.. Abordando a BIM em níveis de modelagem. In: SIMPÓSIO BRASILEIRO DE QUALIDADE DO PROJETO NO AMBIENTE CONSTRUÍDO, IX WORKSHOP BRASILEIRO DE GESTÃO DO PROCESSO DE PROJETO NA CONSTRUÇÃO DE EDIFÍCIOS. São Carlos, 18 a 20 de novembro de 2009.

Scheer, S., Romero, J. Potencial da Implementação da BIM no Processo de Aprovação de Projetos de Edificação na Prefeitura Municipal de Curitiba. Anais do Simpósio Brasileiro de Qualidade do Projeto no Ambiente Construído IX Workshop Brasileiro de Gestão do Processo de Projeto na Construção de Edifícios, 18 a 20 de Novembro de 2009 , São Carlos, SP -PPG-AU EESC USP

Souza, L. L. A., Amorim, S. R. L., Lyrio, A. M. Impactos do uso do BIM em Escritórios de Arquitetura: Oportunidades no Mercado Imobiliário. Gestão \& Tecnologia de Projetos, São Paulo, v.2, no.2, novembro.2009. 